\title{
Tamizaje en las personas mayores con dependencia de cuidados: clave en la estrategia para mitigar la pandemia por COVID-19
}

José F. Parodi * 1,a; Fernando Runzer-Colmenares 1,b; Javier Navarrete-Mejía 2,3,c; Arturo Pareja-Cruz 1,d

\section{RESUMEN}

En la pandemia por la COVID-19, las personas mayores son el grupo que concentra la mayor mortalidad, sobre todo quienes precisan de cuidados de largo plazo por haber perdido su habilidad funcional. Esta población vive en sus domicilios, con la familia o en un centro residencial. Se ha descrito que las personas mayores pueden desarrollar una forma de enfermedad oligosintomática o con una sintomatología clínica particular; por esta razón, las estrategias de tamizaje basadas en síntomas no son las más recomendables. Es necesario detectar de manera precoz a los enfermos en este grupo; por ello, analizamos y proponemos las mejores alternativas disponibles para conseguir este objetivo.

Palabras clave: Anciano; COVID-19; Cuidados de largo plazo (Fuente DeCS BIREME).

\section{Screening for care-dependent older people: a key element in the strategy to mitigate COVID-19 pandemic}

\section{ABSTRACT}

During the COVID-19 pandemic, older people have been the group with the highest mortality rate, especially those who require long-term care for having lost their functional ability. These people are living at home with their family or in a nursing home. It has been described that older people may develop an oligosymptomatic SARS-CoV-2 infection or particular symptoms of the disease. Therefore, symptom-based screening is not the most recommended strategy in this scenario. Since it is necessary to detect early cases in the elderly population, this research work analyzes and proposes the best available alternatives for attaining such goal.

Keywords: Aged; Coronavirus infections; Long-term care (Source: MeSH NLM).

1 Universidad de San Martín de Porres, Facultad de Medicina Humana, Centro de Investigación del Envejecimiento (CIEN). Lima, Perú.

2 Universidad de San Martín de Porres, Facultad de Medicina Humana, Centro de Investigación de Epidemiología Clínica y Medicina Basada En Evidencias (CECLIMBE). Lima, Perú.

3 Universidad Continental. Facultad de Ciencias de la Salud. Lima, Perú.

4 Universidad de San Martín de Porres, Facultad de Medicina Humana, Centro de Investigación de Infectología e Inmunología (CININI).

Lima, Perú.

a Médico Geriatra, MPH, PhD.

b Médico Geriatra, MA, MSc.

c MBA, DPH

d Médico Microbiólogo-Inmunólogo, MPH, PhD.

*Autor corresponsal. 


\section{INTRODUCCIÓN}

La pandemia por COVID-19 ha tenido un impacto devastador en la salud y derechos de las personas mayores

Las publicaciones que han aparecido desde el inicio de la pandemia de la COVID-19 han coincidido en que, de todas las etapas de vida, las personas mayores son quienes han concentrado desproporcionadamente la mortalidad, que ha llegado a ser hasta cinco veces mayor que el promedio mundial en los mayores de 80 años ${ }^{(1)}$. Más del $95 \%$ de las muertes por COVID-19 en Europa y alrededor del $80 \%$ en China han ocurrido en personas mayores de 60 años. En los Estados Unidos, el $80 \%$ de los fallecidos eran adultos mayores de 65 años (2-5). Esta gran letalidad ha sido particularmente elevada en las instituciones de cuidado de largo plazo (ICLP) ${ }^{(6-12)}$.

Es importante resaltar que la COVID-19 no solo impacta en la mortalidad, sino en varios dominios de la capacidad intrínseca (función física, mental, sueño) (13,14), lo que puede cambiar de manera drástica el curso de vida hacia un envejecimiento saludable de las personas ${ }^{(15)}$.

Debido a la gran heterogeneidad en este grupo, existen distintos elementos, como la edad cronológica, que contribuyen a los desenlaces graves. Sin embargo, se mencionan otras características que influirían aún más, como la discapacidad, las comorbilidades y, sobre todo, la fragilidad de las personas mayores y la precariedad de los sistemas de salud para un manejo adecuado; así como las inequidades en el acceso a determinantes sociales de la salud ${ }^{(16-21)}$. Por lo tanto, estrategias de salud para evitar la propagación del coronavirus, y las otras medidas para la optimización funcional en esta población, son imperativas.

\section{ESTRATEGIA DE BÚSQUEDA}

Luego de plantear las preguntas clave para este artículo, se realizó una búsqueda en Pubmed y Liliacs con los siguientes términos: aged, Long-Term, COVID-19. Se incluyeron estudios experimentales, observacionales, guías de práctica clínica y revisiones sistemáticas, con o sin metaanálisis, publicados en el periodo de marzo a agosto del 2020. Los artículos de revisión y reportes de casos fueron excluidos.

\section{El estado y las personas vulnerables en época de pandemia: no existe un grupo poblacional más afectado por las restricciones que el de personas mayores}

Las personas mayores son el grupo con el mayor número de limitaciones en el país. La discriminación por la edad se evidencia en las medidas dictadas por el Gobierno.

A inicios de la pandemia, se estableció la restricción para salir de sus hogares, la política estaba orientada incluso a la prohibición de las visitas a los padres, en especial, los adultos mayores.

En algunos distritos de Lima se prohibió el ingreso de personas mayores de 65 años; y algunos establecimientos de comida y recreación siguieron el mismo ejemplo. La Defensoría del Pueblo, con pronunciamientos constantes, manifiesta lo restrictivo de las libertades de grupos vulnerables, aunque las limitaciones obedecían a disposiciones nacionales.

Abogados de todo el país presentaron en los juzgados pedidos de inconstitucionalidad de las normas dictadas por el Poder Ejecutivo. En ellos se manifiesta que las restricciones son discriminatorias e irrazonables, y más de un especialista solicita el pronunciamiento del Tribunal Constitucional, lo que abre el debate sobre la salud y los derechos fundamentales.

Con el tiempo, el Gobierno se vio obligado a flexibilizar las restricciones, que aún son limitativas para la persona mayor. Estas medidas han generado deterioro en la salud mental, falta de acceso a la atención médica, a los tratamientos y al cobro de pensiones, etc.

Cabe señalar que, pese a las restricciones, las personas mayores dependientes de cuidado se contagiaron y murieron, inclusive sin haber salido nunca de casa, a través de las personas más jóvenes y cuidadores que ingresaron al hogar.

\section{Caso orientador 1}

Carlos es el director de una institución de cuidados a largo plazo, tiene 150 personas mayores con multimorbilidad física y mental que son, en su mayoría, dependientes de cuidados. Él sabe que son la población de mayor mortalidad. Al elaborar su plan de control y prevención de infecciones se encuentra con la disyuntiva sobre el tamizaje periódico para COVID-19, tanto a los residentes como el personal. En su país, las ICLP están bajo responsabilidad directa del Ministerio de Servicios Sociales. Necesita asesoría técnica sobre las siguientes interrogantes: ¿se debe hacer tamizaje para COVID-19 en personal y residentes asintomáticos?, ¿qué tipo de prueba es la que se debe usar?, ¿quién va a pagar estas pruebas? y ¿cómo debe manejar el problema de disminución de cuidadores si estos salen positivos? 
Tamizaje en las personas mayores con dependencia de cuidados: clave en la estrategia para mitigar la pandemia por COVID-19

Las personas mayores con COVID-19 que viven en instituciones de largo plazo concentran la mortalidad y son una pieza clave en la estrategia para mitigar la pandemia

En países desarrollados, la mayoría de los fallecimientos por COVID-19 ocurren en instituciones de cuidado de largo plazo, y pueden constituir entre el 50 al $90 \%$ de todas las muertes por esta enfermedad ${ }^{222}$. El trabajo que se realiza en los ICLP es de altísimo contacto físico cuidador-residente, lo que hace imprescindible una dotación y uso correcto de equipos de protección individual (EPI) para evitar el contagio entre ellos ${ }^{(23,24)}$.

Las condiciones ya descritas y la alta contagiosidad del virus condicionan el alto riesgo en este escenario ${ }^{(25)}$. Pero, además, se ha propuesto que los residentes y trabajadores de la ICLP son los que aceleran la infección, debido a la propagación asintomática ${ }^{(26)}$, e impulsan la epidemia ${ }^{(27) .}$

Las ICLP no pueden seguir siendo un punto ciego para las pruebas prioritarias, el tamizaje y el monitoreo de COVID-19

La detección, aislamiento y tratamiento precoces de los casos de COVID-19 son esenciales para limitar la propagación de la enfermedad en los centros de larga estancia ${ }^{(28)}$.

En muchos países se ha planteado el uso de las pruebas de coronavirus basado en la presencia de síntomas. Sin embargo, está reportado que en las personas que viven o trabajan en una ICLP es imposible determinar con certeza clínica si tienen la enfermedad sin una prueba, que puede dar resultados inexactos, ya que existe una probabilidad mayor de lo normal de obtener resultados falsos negativos ${ }^{(29)}$. De las pruebas realizadas a 98 residentes asintomáticos en una institución, 50 fueron positivos (12). Otro estudio menciona que la transmisión del SARS-CoV-2 entre la población asintomática infectada probablemente contribuyó a la propagación rápida y extensa de la enfermedad a los demás residentes y al personal; y concluye que las estrategias de control de infecciones centradas únicamente en las personas sintomáticas fueron insuficientes para prevenir la transmisión después del ingreso del virus en esa instalación ${ }^{(30)}$.

Las características funcionales y las enfermedades prevalentes de las personas mayores que viven en ICLP hacen que la detección y medidas contra la COVID-19 basadas solamente en el reporte o expresión de los síntomas característicos de la enfermedad sean poco efectivas ${ }^{(12,31)}$. Además, en los mayores, la COVID-19 puede presentarse de manera atípica en personas frágiles o con demencia, como caídas, disfuncionalidad aguda o delirio ${ }^{(32,33)}$.
La gran tasa de casos asintomáticos se ha convertido en el "talón de Aquiles" para el reconocimiento temprano y la acción oportuna para el manejo apropiado de las personas con COVID-19 (34).

Ante esto, algunas organizaciones han recomendado que en ciertas poblaciones, como las personas que viven en establecimientos ICLP, se deben realizar pruebas para la detección del coronavirus de forma masiva y no solo a las personas con síntomas ${ }^{(35-37)}$.

Según el método Delphi, un panel de expertos en medicina de cuidados de largo plazo recomienda el uso adecuado de equipos de protección personal (EPP) y cumplir las siguientes acciones:

- Si las pruebas RT-PCR están disponibles, emplearlas para los residentes y el personal, y enfatizar la importancia de contar con ellas.

- Evaluar a los residentes con síntomas atípicos en un escenario de pruebas limitadas.

- Repetir las pruebas cada una o dos semanas, si están disponibles, y reducir la frecuencia cada mes, a medida que disminuye la prevalencia comunitaria, o cuando la recopilación de datos adicionales así lo indique.

El mismo grupo de expertos reconoció que las pruebas frecuentes tendrían consecuencias como la escasez potencial de personal (debido a la cuarentena que se debe seguir luego de tener una prueba positiva) y el uso más frecuente de EPP. Sin embargo, el panel consideró si no se toman las pruebas aparecerían nuevos grupos de infectados y que la alta tasa de mortalidad resultante superaría las posibles consecuencias negativas de las pruebas ${ }^{(26)}$.

\section{La selección de la prueba adecuada para contribuir a la mitigación de la COVID-19}

Muchas organizaciones brindan orientación sobre quién debe hacerse la prueba, pero también señalan que las decisiones sobre la prueba las toman los departamentos de salud o los proveedores de atención médica del estado y del lugar. Al mismo tiempo mencionan que en las ICLP se deben realizar las llamadas pruebas virales de detección de antígeno o ácido ribonucleico (ARN) ${ }^{(37)}$. Dado que la prueba de detección de antígenos no está disponible, la prueba de detección de ARN viral más confiable y de uso más común para el diagnóstico de COVID-19 es la prueba de RT-PCR realizada con hisopados nasofaríngeos u otras muestras del tracto respiratorio superior, como hisopados de garganta o muestras de saliva ${ }^{(38)}$.

Respecto a las pruebas de anticuerpos, una revisión actualizada menciona que tienen una sensibilidad muy baja en la primera semana (desde el inicio de los síntomas) para ser importantes en el diagnóstico de la COVID-19. Además, 
se indica que la sensibilidad se ha evaluado principalmente en pacientes hospitalizados, por lo que no está claro si las pruebas detectarían niveles de anticuerpos más bajos, como ocurriría en las formas leve y asintomática de la COVID-19 ${ }^{(39)}$.

Las pruebas tipo inmunoensayo para la detección de anticuerpos (Ac) específicos contra antígenos del SARS-CoV-2 son diversas. En esta diversidad influyen de manera significativa el fundamento de la técnica elegida, el anticuerpo que se detecta y la estrategia empleada para la formación del complejo antígenoanticuerpo. Los inmunoensayos de flujo lateral son muy utilizados para detectar Ac IgM e IgG contra SARS-CoV-2. Además, estas pruebas no cuantifican la presencia de los Ac específicos contra el virus. Diversas plataformas han desarrollado herramientas complejas de diagnóstico y seguimiento para detectar al nuevo coronavirus y muestran gran variabilidad en su aplicación, como las pruebas indirectas para identificar anticuerpos antiSARS COV-2. En este contexto, aparecen nuevas técnicas inmunológicas, como el inmunoensayo magnético con soporte de nanopartículas, que es una prueba serológica cuantitativa que permite identificar anticuerpos IgM, IgG e IgA, cuya variante puede detectar anticuerpos anti-SARS-CoV-2 ${ }^{(40)}$.

En muchos países, la poca cantidad de pruebas existentes se ha empleado en los hospitales, por lo que las ICLP han afrontado dificultades serias para la detección precoz de los enfermos. A esto se suma la falta de acreditación de calidad de las distintas técnicas disponibles. Otro reto es el financiamiento sostenible para las estrategias basadas en la toma sistemática de pruebas. Respecto al tiempo para obtener el resultado, la prueba de antígeno es más rápida que la molecular. La figura 1 muestra las consideraciones para la interpretación exámenes moleculares y de antígeno en las ICLP.

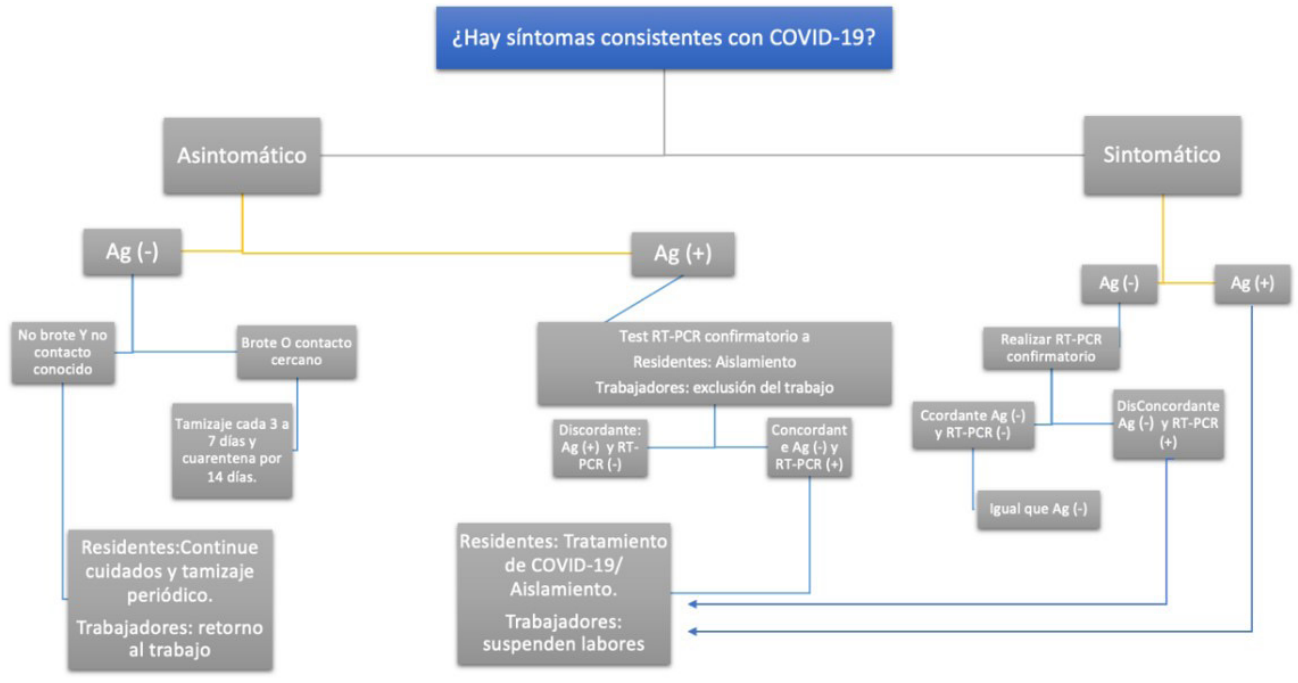

Figura 1. Consideraciones para la interpretación de pruebas de tamizaje en ICLP

Las personas asintomáticas con resultado positivo en la prueba de antígeno pueden no necesitar pruebas de confirmación si tienen probabilidad previa a la prueba (por ejemplo, una persona residía con otra persona infectada en una instalación de un gran brote, como con prevalencia mayor a $20 \%$ ).

Ag = antígeno

RT-PCR = prueba de amplificación de ácido nucleico, incluida la reacción en cadena de la polimerasa con transcriptasa (44)

Ante esto surgen preguntas respecto a la priorización o equilibrio de la inversión en salud durante la pandemia; por ejemplo, decidir si comprar más ventiladores mecánicos para los hospitales o comprar pruebas para la detección precoz en las ICLP. Algunos autores intentan dar una respuesta y han apelado a la frase "Mientras esperamos lo mejor, debemos prepararnos para lo peor frenando la propagación" (41).
Para seleccionar las pruebas más convenientes es necesario considerar sus sensibilidades y especificidades, y también los valores predictivos que dependen de la prevalencia (se asume que está entre 60 y $80 \%$ en los ICLP) y que son mucho más importantes ${ }^{(25)}$. En ese sentido, la PCR tiene una sensibilidad de alrededor del 80 \% (según la técnica utilizada) y una especificidad cercana al $100 \%$. 
Tamizaje en las personas mayores con dependencia de cuidados: clave en la estrategia para mitigar la pandemia por COVID-19

\section{Caso orientador 2}

Cristina es una mujer de 98 años, lúcida, pero con limitaciones de movilidad, por lo que requiere una cuidadora para su subsistencia básica y realización de trámites. Vive con su hija de 77 años y con su yerno de 78 que aún trabaja como médico. Cristina tiene 2 cuidadoras contratadas que hacen turnos de 12 horas para atenderla. Los primeros días de la pandemia las cuidadoras no estuvieron, pero es necesario que vuelvan a su trabajo. Además del uso apropiado del EPP y distanciamiento social durante sus salidas diarias, ¿es recomendable que se hagan pruebas periódicas para tamizaje de COVID-19? y ¿qué pruebas deben hacerse y con qué frecuencia?

\section{¿Se deben extender las recomendaciones sobre el uso de pruebas virales a las personas dependientes de cuidados que viven en sus casas y a sus cuidadores?}

Aún faltan datos sobre la mortalidad y deterioro funcional de las personas mayores dependientes de cuidado en los domicilios. En este grupo se ha reportado un aumento del estrés de los cuidadores, una sensación de falta de eficacia para el cuidado, la deserción laboral de los cuidadores formales ${ }^{(42)}$ y una espiral de fragilidad como resultado de la pandemia de COVID-19 ${ }^{(43)}$.

Teniendo en cuenta el riesgo y las características clínicas similares, surge la pregunta acerca de si se debe extrapolar la recomendación de tomar las pruebas virales de manera sistemática a las personas mayores dependientes de cuidado que viven en su casa y a sus cuidadores.

\section{CONCLUSIONES}

Según la literatura consultada, es evidente que para las autoridades de salud de los países de América Latina y el Caribe son necesarias recomendaciones y acciones claras y adecuadas sobre la pertinencia y modalidad de toma de muestras para tamizaje de coronavirus en personas mayores dependientes de cuidado y sus cuidadores en las instituciones de cuidado de largo plazo y en sus domicilios. De esta manera, tendrían un instrumento técnico para mejorar su rol asesor y rector en materia de salud en estos escenarios. Esta recomendación pretende optimizar las otras medidas para mitigar la pandemia por coronavirus como el uso adecuado de EPP, el distanciamiento social y la gestión de la atención adecuada de los casos detectados.

Contribución de los autores: José F. Parodi contribuyó con la concepción, diseño y redacción del primer y los siguientes borradores del documento. Javier Navarrete, Arturo Pareja y Fernando Runzer han contribuido con la revisión bibliográfica y la redacción del trabajo.

\section{Fuentes de financiamiento: Este artículo ha sido financiado} por los autores.

Conflictos de interés: Los autores declaran no tener ningún conflicto de interés.

\section{REFERENCIAS BIBLIOGRÁFICAS}

1. World Health Organization. COVID-19 Strategy Update [Internet]. WHO; 2020. Disponible en: https://www.who.int/publications-detail/covid19-strategy-update---14-april-2020

2. Zhou F, Yu T, Du R, Fan G, Liu Y, Liu Z, et al. Clinical course and risk factors for mortality of adult inpatients with COVID-19 in Wuhan, China: a retrospective cohort study. Lancet. 2020; 395(10229): 1054-62.

3. Guan WJ, Ni ZY, Hu Y, Liang WH, Ou CQ, He JX, et al. Clinical Characteristics of Coronavirus Disease 2019 in China. N Engl J Med. 2020; 382: $1708-20$.

4. Kluge H. Statement - Older people are at highest risk from COVID-19, but all must act to prevent community spread [Internet]. World Health Organization; 2020. Disponible en: https://www.euro.who.int/en/ health-topics/health-emergencies/coronavirus-covid-19/statements/ statement-older-people-are-at-highest-risk-from-covid-19,-but-allmust-act-to-prevent-community-spread

5. Bialek S, Boundy E, Bowen V, Chow N, Cohn A, Dowling N, et al. Severe outcomes among patients with coronavirus disease 2019 (COVID-19) United States, February 12-March 16, 2020. MMWR Morb Mortal Wkly Rep. 2020; 69(12): 343-6.

6. McMichael TM, Clark S, Pogosjans S, Kay M, Lewis J, Baer A, et al. COVID-19 in a long-term care facility - King county, Washington, February 27-March 9, 2020. MMWR Morb Mortal Wkly Rep. 2020; 69(12): 339-42.

7. Lorenz-Dant K. Germany and the COVID-19 long-term care situation. International Long-Term Care Policy Network. 2020.

8. D'Adamo H, Yoshikawa T, Ouslander JG. Coronavirus Disease 2019 in Geriatrics and Long-Term Care: The ABCDs of COVID-19. J Am Geriatr Soc. 2020; 68(5): 912-7.

9. Low LF. The Long-Term Care COVID-19 situation in Australia. 2020; (April): 1-5.

10. Lai CC, Wang JH, Ko WC, Yen MY, Lu MC, Lee CM, et al. COVID-19 in long-term care facilities: An upcoming threat that cannot be ignored. J Microbiol Immunol Infect. 2020; 53(3): 444-6.

11. Comas-Herrera A, Zalakain J. Mortality associated with COVID-19 outbreaks in care homes: early international evidence. Resources to support community and institutional Long-Term Care responses to COVID-19. 2020; (April): 1-6.

12. American Geriatrics Society. American Geriatrics Society Policy Brief: COVID-19 and Nursing Homes. J Am Geriatr Soc. 2020; 68(5): 908-11.

13. Yamada $M$, Kimura $Y$, Ishiyama D, Otobe $Y$, Suzuki M, Koyama S, et al. Effect of the COVID-19 Epidemic on Physical Activity in CommunityDwelling Older Adults in Japan: A Cross-Sectional Online Survey. J Nutr Health Aging. 2020; 24(9): 948-50.

14. Sepúlveda-Loyola W, Rodríguez-Sánchez I, Pérez-Rodríguez P, Ganz F, Torralba R, Oliveira DV, et al. Impact of Social Isolation Due to COVID-19 on Health in Older People: Mental and Physical Effects and Recommendations. J Nutr Health Aging. 2020: 1-10.

15. Chakraborty A, Ghosh S, Mukhopadhyay P, Dinara SM, Bag A, Mahata MK, et al. Trapping effect analysis of AlGaN/InGaN/GaN Heterostructure by conductance frequency measurement. MRS Proceedings. 2014; 2: 81-7. 
16. Bello-Chavolla OY, González-Díaz A, Antonio-Villa NE, Fermín-Martínez CA, Márquez-Salinas A, Vargas-Vázquez A, et al. Unequal impact of structural health determinants and comorbidity on COVID-19 severity and lethality in older Mexican adults: Looking beyond chronological aging. J Gerontol A Biol Sci Med Sci. 2020: glaa163.

17. Nikolich-Zugich J, Knox KS, Rios CT, Natt B, Bhattacharya D, Fain MJ. SARS-CoV-2 and COVID-19 in older adults: what we may expect regarding pathogenesis, immune responses, and outcomes. GeroScience. 2020; 42(2): 505-14.

18. Canada.ca. Infection Prevention and Control for COVID-19: Interim Guidance for Long Term Care Homes [Internet]. Government of Canada; 2020. Disponible en: https://www.canada.ca/ en/public-health/services/diseases/2019-novel-coronavirusinfection/prevent-control-covid-19-long-term-care-homes. html\#a5\%0Ahttps://www.canada.ca/en/public-health/services/ diseases/2019-novel-coronavirus-infection/prevent-control-cov

19. Cesari M, Marzetti E, Thiem U, Pérez-Zepeda MU, Abellan Van Kan G, Landi $F$, et al. The geriatric management of frailty as paradigm of the end of the disease era. Eur J Intern Med. 2016; 31: 11-4.

20. Volpato S, Landi F, Incalzi RA. A Frail Health Care System for an Old Population: Lesson form the COVID-19 Outbreak in Italy. J Gerontol A Biol Sci Med Sci. 2020; 75(9): e126-7.

21. Landi F, Barillaro C, Bellieni A, Brandi V, Carfi A, D'Angelo M, et al. The New Challenge of Geriatrics: Saving Frail Older People from the SARSCoV-2 Pandemic Infection. J Nutr Health Aging. 2020; 24(5): 466-70.

22. Lau-Ng R, Caruso LB, Perls TT. COVID-19 Deaths in Long-Term Care Facilities: A Critical Piece of the Pandemic Puzzle. J Am Geriatr Soc. 2020; 17(11): jgs.16669.

23. Sociedad Española de Geriatría y Gerontología. La SEMEG y la SEGG quieren hacer pública su posición sobre el tratamiento de la epidemia de Coronavirus en residencias de personas mayores [Internet] 2020. Disponible en: https://www.semeg.es/semeg-institucional/ actualidad/_/la-semeg-y-la-segg-quieren-hacer-publica-suposicion-sobre-el-tratamiento-de-la-epidemia-de-coronavirus-enresidencias-de-personas-mayores-.html

24. Ouslander JG. Coronavirus Disease19 in Geriatrics and Long-Term Care: An Update. J Am Geriatr Soc. 2020; 68(5): 918-21.

25. Getafe SG. Plan de Coordinacion y apoyo a las Residencias. Servicio de Geriatría Hospital Universitario de Getafe. 2020.

26. Wasserman M, Ouslander JG, Lam A, Wolk AG, Morley JE, PreyssFriedman SV, et al. Diagnostic Testing for SARS-Coronavirus-2 in the Nursing Facility: Recommendations of a Delphi Panel of Long-Term Care Clinicians. J Nutr Health Aging. 2020: 1-6.

27. Li R, Pei S, Chen B, Song Y, Zhang T, Yang W, et al. Substantial undocumented infection facilitates the rapid dissemination of novel coronavirus (SARS-CoV-2). Science. 2020; 368(6490): 489-93.

28. Organización Mundial de la Salud. Prevención y control de infecciones en los centros de atención de larga estancia en el contexto de la COVID-19: Orientaciones Provisionales [Internet]. 2020. Disponible en:https: / /www.paho.org/es/documentos/prevencion-controlinfecciones-centros-atencion-larga-estancia-contexto-covid-19-0

29. Jha S. False Negative: COVID-19 Testing's Catch-22 [Internet]. MedPage Today. 2020. Disponible en: https://www.medpagetoday. com/infectiousdisease/covid19/85717

30. Arons MM, Hatfield KM, Reddy SC, Kimball A, James A, Jacobs JR, et al. Presymptomatic SARS-CoV-2 Infections and Transmission in a Skilled Nursing Facility. N Engl J Med. 2020; 382(22): 2081-90.

31. Comas-Herrera A. The problem of asymptomatic COVID-19 infections among care home staff and residents: emerging evidence and implications. LTC COVID. 2020; (April): 1-5.

32. Tay HS, Harwood R. Atypical presentation of COVID-19 in a frail older person. Age Ageing. 2020; 49(4): 523-4.

33. Bianchetti A, Rozzini R, Guerini F, Boffelli S, Ranieri P, Minelli G, et al. Clinical Presentation of COVID19 in Dementia Patients. J Nutr Health Aging. 2020; 24(6): 560-2.

34. Gandhi M, Yokoe DS, Havlir D v. Asymptomatic transmission, the achilles' heel of current strategies to control Covid-19. N Engl J Med. 2020; 382(22): 2158-60.

35. Centers for Disease Control and Prevention. Infection Control for Nursing Homes [Internet]. 2020. Disponible en: https://www.cdc. gov/coronavirus/2019-ncov/hcp/long-term-care.html

36. Centers for Disease Control and Prevention. Testing Guidelines for Nursing [Internet]. 2020. Disponible en: https://www.cdc.gov/ coronavirus/2019-ncov/hcp/nursing-homes-testing.html

37. Centers for Disease Control and Prevention. Overview of Testing for SARS-CoV-2 [Internet]. 2020. Disponible en: https://www. cdc.gov/coronavirus/2019-ncov/hcp/testing-overview.html?CDC_ AA_refVal=https $\% 3 A \% 2 F \% 2 F w w w . c d c . g o v \% 2 F$ coronavirus $\% 2 F 2019$ ncov\%2Fhcp\%2Fclinical-criteria.html

38. Sethuraman N, Jeremiah SS, Ryo A. Interpreting Diagnostic Tests for SARS-CoV-2. JAMA. 2020; 323(22): 2249-51.

39. Deeks JJ, Dinnes J, Takwoingi Y, Davenport C, Spijker R, TaylorPhillips $S$, et al. Antibody tests for identification of current and past infection with SARS-CoV-2. Cochrane Database Syst Rev. 2020; 2020(6): CD013652.

40. Pareja Cruz A, Lizaraso-Soto FA. Un nuevo coronavirus, una nueva enfermedad COVID-19. Primera. Universidad de San Martin de Porres, editor. Lima, Peru; 2020.

41. Halpern SD, Truog RD, Miller FG. Cognitive Bias and Public Health Policy During the COVID-19 Pandemic. JAMA. 2020; 324(4): 337-8.

42. Navarrete-Mejía PJ, Rivera-Encinas MT, Runzer-Colmenares FM, Velasco-Guerrero JC, Sallcahuaman-Valdiglesias E. Profile of the senior caregiver in situation of pandemic for SARS-COV-2. Rev Cuerpo Méd HNAAA. 2020; 13(1): 26-31.

43. Abbatecola AM, Antonelli-Incalzi R. COVID-19 Spiraling of Frailty in Older Italian Patients. J Nutr Health Aging. 2020; 24(5): 453-5.

44. Centers for Disease Control and Prevention. Considerations for Interpretation of Antigen Tests in Long-Term Care Facilities [Internet]. 2021. Disponible en: https://www.cdc.gov/coronavirus/2019-ncov/ downloads/hcp/nursing-home-testing-algorithm-508.pdf

\section{Correspondencia:}

José Francisco Parodi García

Alameda del Corregidor cuadra 15, Urb. Las Viñas, La Molina. Lima, Perú.

Teléfono: +51998139858

Correo electrónico: jparodig@usmp.pe

Recibido: 12 de marzo de 2021

Evaluado: 26 de marzo de 2021 Aprobado: 18 de abril de 2021

(c) La revista. Publicado por Universidad de San Martín de Porres, Perú. (cc) bajo términos de Licencia Creative Commons Atribución 4.0 Internacional. (http://creativecommons.org/licenses/by/4.0/)

\section{ORCID iDs}

José F. Parodi

Javier Navarrete Mejía

Arturo Pareja Cruz

(1) https: / / orcid.org/0000-0002-0336-0584

C https: / /orcid.org/0000-0002-9809-6789

Fernando Runzer Colmenares [D https: / / orcid.org/0000-0002-7717-8996 